\title{
COOPERATIVE SYSTEMS WITH ANY NUMBER OF SPECIES
}

\author{
$\mathrm{BY}$ \\ MANUEL DELGADO AND ANTONIO SUÁREZ
}

\begin{abstract}
Departamento de Ecuaciones Diferenciales y Análisis Numérico, c/ Tarfia, s/n, Universidad de Sevilla, 41012-Sevilla
\end{abstract}

\begin{abstract}
In this paper we study the positive solutions of a cooperative system of any number of equations which consider the case of the slow diffusion and include the Lotka-Volterra model. We determine conditions of existence of global solution and blow-up in finite time in terms of the value of the spectral radius of a certain nonnegative matrix associated to the system. The results generalize the ones known for the particular case of two equations and we justify them by using the specific properties of nonnegative matrices which translate the cooperative character of the system.
\end{abstract}

1. Introduction. Let $\Omega \subset \mathbf{R}^{N}$ be a regular domain with $N \geq 1$. For each $T>0$, we denote $D_{T}=(0, T] \times \Omega, S_{T}=(0, T] \times \partial \Omega$, and consider

$$
\left\{\begin{array}{llll}
\frac{\partial}{\partial t} w_{i}-\Delta\left(w_{i}^{m}\right) & =w_{i}\left(\lambda_{i}-w_{i}+\sum_{\substack{j=1 \\
j \neq i}}^{n} a_{i j} w_{j}\right) & & \text { in } D_{T}, \\
w_{i} & =0 & \text { in } S_{T}, & \\
w_{i}(0, x) & =w_{i}^{0}(x) & \text { in } \bar{\Omega} .
\end{array}\right.
$$

This problem can be viewed as a model for the temporal evolution of the population density of $n$ species which cooperate two against two, confined in a domain $\Omega$ in which boundary they can not live and which spread slowly. Here, $\lambda_{i} \in \mathbf{R}$ are the birth rates (if positive) or the death rates (if negative) of each species, $m>1$ is the parameter which entails the diffusion rate (the diffusion decreases when $m$ increases) and $a_{i j}>0$ describe the interaction rates of the species.

In the particular case $n=2$, in [2] the existence of positive global solution in time of (1) is proved assuming the existence of a pair of sub-supersolutions of (1).

Received October 3, 2001.

2000 Mathematics Subject Classification. Primary 35K50, 35K57.

This work was supported by CICYT of Spain under grant MAR98-0486 and BFM2000-0797.

E-mail address: delgado@numer.us.es

E-mail address: suarez@numer.us.es

(C)2003 Brown University 
In this paper, we consider the following system:

$$
\left\{\begin{array}{llll}
\frac{\partial}{\partial t} u_{i}-\Delta u_{i} & =u_{i}^{1 / m}\left(\lambda_{i}-u_{i}^{1 / m}+\sum_{\substack{j=1 \\
j \neq i}}^{n} a_{i j} u_{j}^{1 / m}\right) & & \text { in } D_{T}, \\
u_{i} & =0 & & \text { in } S_{T}, \\
u_{i}(0, x) & =u_{i}^{0}(x) & \text { in } \bar{\Omega} .
\end{array}\right.
$$

Problems (1) and (2) are different but related systems; for instance, in [4] for $n=2$ it is proved that its linearizing at the steady-state are equivalent.

The case of two species (i.e., $n=2$ ) was approached in [6] when $m=1$ and in [4] when $m>1$ where we turn for more details about the genesis of the model. The results of the papers show the great difference between the two cases. The case $n=3$ was studied in [5]. In the case $n=2$ and $m>1$ we obtained the following result:

Theorem 1.1. (1) Suppose $a_{12} a_{21}<1$. If $\left(\lambda_{1}, \lambda_{2}\right) \in \mathbf{R}_{+}^{2} \backslash\{(0,0)\}$, then problem (2) has one unique positive global solution $\left(u_{1}(t, x), u_{2}(t, x)\right)$ and the corresponding steady problem has at least one positive solution $\left(u_{1 s}(x), u_{2 s}(x)\right)$. Moreover, if one of the following hypotheses is satisfied:

- $1<m<2$ and $a_{12}$ and $a_{21}$ "sufficiently small" (cf. [4] for specific bounds),

- $m=2$,

- $2<m, 1-a_{12} a_{21}(m-1)^{2}>0, \lambda\left(1-a_{12} a_{21}(m-1)\right) \geq a_{12} \mu(m-2)$, $\mu\left(1-a_{12} a_{21}(m-1)\right) \geq a_{21} \lambda(m-2)$,

then, the positive solution of the steady problem is unique.

(2) Suppose $a_{12} a_{21}=1$. If $m>1$ and $\left(\lambda_{1}, \lambda_{2}\right) \in \mathbf{R}_{+}^{2} \backslash\{(0,0)\}$, then there exists one unique positive global solution $\left(u_{1}(t, x), u_{2}(t, x)\right)$ and the corresponding steady problem has at least one positive solution.

(3) Suppose $a_{12} a_{21}>1$.

(a) If $1<m<2$, there exist $0<\underline{\lambda_{i}}<\overline{\lambda_{i}}$, for $i=1,2$ such that for $\lambda_{i}>\overline{\lambda_{i}}$ the solution of $(2)\left(u_{1}(t, x), u_{2}(t, \overline{x)})\right.$ blows up in finite time and if $0<\lambda<\underline{\lambda_{i}}$, then (2) has positive global solution and the corresponding steady problem has at least one positive solution.

(b) If $m=2$, problem (2) has positive global solution which can be not bounded.

(c) If $2<m$ and $\left(\lambda_{1}, \lambda_{2}\right) \in \mathbf{R}^{2} \backslash\{(0,0)\}$, then (2) has positive global solution and the corresponding steady problem has at least a positive solution.

As a consequence, the behaviour of the solutions of the problem depends crucially on the product $a_{12} a_{21}$ whose value $>1$ or $<1$ determines the so-called strong or weak interactions in the literature for the case $m=1$ and $n=2$ (cf., for instance, [8] and [3]). In [5] we justified that if we introduce the third equation, the spectral radius of certain matrix is the parameter which determines the behaviour of the solutions. However, there we used specific arguments for matrix of order 3 which cannot be adapted to cover the general case. In this paper we will prove a result for a system of $n$ equations where the importance of the cooperative character of the system will be very clear. We will limit our framework to the case $\lambda_{i}>0, \quad i=1, \ldots, n$, although results can be given in other cases. To our knowledge, the results are new even in the linear case $(m=1)$. 
We will call the matrix of the system (2)

$$
\mathcal{A}=\left(\begin{array}{cccc}
-1 & a_{12} & \ldots & a_{1 n} \\
a_{21} & -1 & \ldots & a_{2 n} \\
\vdots & \vdots & \ddots & \vdots \\
a_{n 1} & a_{n 2} & \ldots & -1
\end{array}\right) .
$$

Associated to $\mathcal{A}$, we consider the matrix

$$
A=\left(\begin{array}{cccc}
0 & a_{12} & \ldots & a_{1 n} \\
a_{21} & 0 & \ldots & a_{2 n} \\
\vdots & \vdots & \ddots & \vdots \\
a_{n 1} & a_{n 2} & \ldots & 0
\end{array}\right)
$$

that will be transformed into our object of study. Note that in a general case, when the matrix of the system is

$$
\mathcal{A}=\left(\begin{array}{cccc}
-a_{11} & a_{12} & \ldots & a_{1 n} \\
a_{21} & -a_{22} & \ldots & a_{2 n} \\
\vdots & \vdots & \ddots & \vdots \\
a_{n 1} & a_{n 2} & \ldots & -a_{n n}
\end{array}\right)
$$

then the problem can be reduced to the previous form, if $m \neq 2$, through a linear variable change which provides us the associated matrix

$$
A=\left(\begin{array}{cccc}
0 & b_{12} & \ldots & b_{1 n} \\
b_{21} & 0 & \ldots & b_{2 n} \\
\vdots & \vdots & \ddots & \vdots \\
b_{n 1} & b_{n 2} & \ldots & 0
\end{array}\right)
$$

being

$$
b_{i j}=a_{i j} a_{i i}^{(m-1) /(2-m)} a_{j j}^{1 /(m-2)} .
$$

This transformation also changes the birth rates, but this is not outstanding in the following.

The outline of the paper is as follows. In Sec. 2, we recall some results from the theory of matrices that we will need. In Sec. 3 we extend the results about the subsupersolution method, the sweeping principle, and the uniqueness of positive solution of parabolic problem to systems with more than two equations. In Sec. 4 we carry out the estimates for the steady problem. In the following section we study the parabolic problem in the different cases. In the last section we will give a summary of results in the case $m=1$.

2. Some results from the theory of matrices. Consider a matrix $M$ of nonnegative elements and dimension $s$; let $\rho(M)$ denote its spectral radius. It is well known, by Perron-Frobenius theorem (cf. [11]), that $M$ has a real positive eigenvalue, $\bar{\rho}$, such that $\rho(M)=\bar{\rho}$. We denote by

$$
\phi_{M}(\lambda)=\operatorname{det}(\lambda I-M)
$$


which is a polynomial with positive main coefficient. It is clear that if $\phi_{M}(1)<0$, then there exists a real eigenvalue greater than 1 . So

$$
\phi_{M}(1)=\operatorname{det}(I-M)<0 \Longrightarrow \rho(M)>1 .
$$

On the other hand, we recall that a principal minor of a matrix $M$ is the determinant of a square submatrix of $M$ obtained by crossing out any $j$ rows and the corresponding $j$ columns of $M$, where $1 \leq j \leq n$. It is also well known (cf. [1], pg. 150, for instance) that for $k=1, \ldots, s-1, \phi_{M}^{k)}(1)$ is equal to the sum of the principal minors of dimension $s-k$ multiplied by $k$ !. So, if all of the principal minors of order $s-k$ of $M$ are nonnegative, then $\phi_{M}^{k)}(1) \geq 0$.

LEMMA 2.1. If all of the principal minors of $M$ are nonnegative and $\rho(M)>1$, then $\operatorname{det}(I-M)<0$.

Proof. The Taylor formula allows us to write

$$
\phi_{M}(\lambda)=\phi_{M}(1)+\phi_{M}^{\prime}(1)(\lambda-1)+\ldots+\frac{\phi_{M}^{s)}(1)}{s !}(\lambda-1)^{s} .
$$

By hypotheses, $\phi_{M}^{\prime}(1), \ldots, \phi_{M}^{s-1)}(1)$ are nonnegative and $\phi_{M}^{s)}(1)=s !>0$; therefore, since $\phi_{M}(1) \geq 0$, it follows that $\phi_{M}(\lambda)>0, \quad \forall \lambda>1$. So $\rho(M) \leq 1$.

Finally, we turn to [11] for the definition of irreducible matrix. Note that a matrix as $A$ is irreducible. We will use the following result (cf. [11], Lemma 2.6).

THEOREM 2.2. If $M$ is an irreducible matrix of nonnegative terms and $M_{i}$ is a principal matrix of $M$, then $\rho\left(M_{i}\right)<\rho(M)$.

3. Preliminaries. We will collect some results that will be used throughout this paper. Consider the matrix operator $\mathcal{L}=\operatorname{diag}(-\Delta, \ldots,-\Delta)$. We pose

$$
f(x, z): \bar{\Omega} \times \mathbf{R}_{+}^{n} \mapsto \mathbf{R}^{n}, f \in \mathcal{F}, f \text { increasing in } z,
$$

where $\mathcal{F}:=C^{\beta}\left(\bar{\Omega} \times \mathbf{R}_{+}^{n} ; \mathbf{R}^{n}\right) \cap C^{2}\left(\Omega \times(0, \infty)^{n} ; \mathbf{R}^{n}\right), \beta \in(0,1) . f$ is called increasing in $z$ if $f_{i}$ is increasing in $z_{j}$ for all $j \neq i$.

Consider the following parabolic system

$$
\begin{cases}z_{t}+\mathcal{L} z=f(x, z) & \text { in } D_{T} \\ z=0 & \text { on } S_{T} \\ z(0, x)=z_{0}(x) & \text { in } \bar{\Omega}\end{cases}
$$

where $z_{0} \in\left(C^{\beta}(\bar{\Omega})\right)^{n}$ and satisfies the compatibility condition $z_{0}(x)=0$ on $\partial \Omega$.

Definition 3.1. Suppose $(H R)$. We will say that $\underline{z}, \bar{z} \in\left(C^{\beta}\left(\overline{D_{T}}\right) \cap C^{2}\left(D_{T}\right)\right)^{n}$ is a sub-supersolution of (5) if $\underline{z} \leq \bar{z}$ in $D_{T}$ and:

(1) $\bar{z}_{t}+\mathcal{L} \bar{z}-f(x, \bar{z}) \geq 0 \geq \underline{z}_{t}+\mathcal{L} \underline{z}-f(x, \underline{z})$ in $D_{T}$,

(2) $\bar{z} \geq 0 \geq \underline{z}$ on $S_{T}$,

(3) $\bar{z}(0, x) \geq z_{0}(x) \geq \underline{z}(0, x)$ in $\bar{\Omega}$.

We have (cf. [4]) 
Theorem 3.2. Suppose $(H R)$ and that there exists a sub-supersolution $\underline{z}, \bar{z}$ of $(5)$. Then there exist $z_{*}$ and $z^{*} \in\left(C^{1+\beta}\left(\overline{D_{T}}\right) \cap C^{2+\beta}\left(D_{T}\right)\right)^{n}$ which are minimal and maximal solutions of (5), respectively, in the sense that for every solution $z \in\left(C^{1+\beta}\left(\overline{D_{T}}\right) \cap\right.$ $\left.C^{2+\beta}\left(D_{T}\right)\right)^{n}$ of $(5)$ with $\underline{z} \leq z \leq \bar{z}$, it is verified that $z_{*} \leq z \leq z^{*}$.

For the uniqueness of positive solution of (5), we need some notation. We denote by

$$
\operatorname{int}(P):=\left\{u \in C_{0}^{1}(\bar{\Omega}): u(x)>0 \text { in } \Omega \text { and } \partial u / \partial n<0 \text { on } \partial \Omega\right\},
$$

where $n$ stands for the outward unit normal on $\partial \Omega$. We say that a function $u \in C_{0}^{1}(\bar{\Omega})$ is positive if $u \in \operatorname{int}(P)$. Let $M(x)=\left(m_{i j}(x)\right)$ be a matrix of order $n \times n$ whose elements $m_{i j} \in C^{1}(\Omega)$ verify

$$
\left\{\begin{array}{l}
m_{i j}>0 \quad i \neq j, \\
\exists K>0, \alpha \in[0,2) \text { such that }\left|m_{i j}(x)\right|[\operatorname{dist}(x, \partial \Omega)]^{\alpha} \leq K .
\end{array}\right.
$$

The following result was proved in [4] in the case $n=2$ and the proof extends easily to the general case.

Proposition 3.3. Suppose $(H R)$ and that for every $z \in(\operatorname{int}(P))^{n}$, the matrix

$$
D F(z)(x):=\left(D_{i} f_{j}(x, z(x))\right)
$$

satisfies $(H M)$. Then there exists at most one positive solution, i.e., $z(t, x) \in(\text { int }(P))^{n}$ for any $t \geq 0$, of $(5)$.

In order to obtain a priori bounds of the stationary solutions of (5) and some uniqueness results, we will use the following sweeping principle for systems (see Lemma 3 in [4] and Theorem 4 in [9]).

Proposition 3.4. Suppose $(H R)$ and that $f$ satisfies the hypotheses of Proposition 3.3. Consider $z$ a steady solution of (5) and $z_{r}$ with $r \in\left(r_{0}, r_{1}\right]$ a family of positive functions such that:

(1) $\mathcal{L} z_{r} \geq f\left(x, z_{r}\right)$ in $\Omega$ and $z_{r} \geq 0$ on $\partial \Omega$,

(2) $z_{r}$ depends on $r$ continuously and is increasing on $r$ for all $x \in \Omega$,

(3) $z \leq z_{r_{1}}$ and $z \neq z_{r}$ for all $r$,

(4) Either $z_{r}$ is strictly increasing on $r$ or $\partial z_{r} / \partial n$ depends continuously on $r$ for $x \in \partial \Omega$.

Then,

$$
z \leq \inf _{r \in\left(r_{0}, r_{1}\right]} z_{r}
$$

REMARK 3.5. The same result is true for a family of subsolutions, with the appropriate changes in the inequalities.

4. A priori estimates for the solutions of the steady system. We consider in this section the steady system corresponding to (2), i.e.,

$$
\left\{\begin{aligned}
-\Delta u_{i} & =u_{i}^{1 / m}\left(\lambda_{i}-u_{i}^{1 / m}+\sum_{\substack{j=1 \\
j \neq i}}^{n} a_{i j} u_{j}^{1 / m}\right) & & \text { in } \Omega, \\
u_{i}=0 & & \text { on } \partial \Omega . & 1 \leq i \leq n
\end{aligned}\right.
$$


The following result holds:

THEOREM 4.1. We define

$$
b_{i i}=0, \quad b_{i j}=a_{i j} \quad \text { for } i \neq j, \quad 1 \leq i, j \leq n
$$

and let

$$
A=\left(b_{i j}\right) .
$$

We denote $\alpha=\min _{1 \leq i \leq n} \lambda_{i}$ and $\beta=\max _{1 \leq i \leq n} \lambda_{i}$. If $\rho(A)<1$ and $\alpha>0$, then there exist $n+1$ positive constants $P_{i}, i=1, \ldots, n$ and $r_{0}$ such that any positive solution, $u_{i}$, of (6) verifies:

(1) If $1<m<2$,

$$
\theta_{\alpha} \leq u_{i} \leq r_{0} P_{i} \theta_{\beta}, \quad i=1, \ldots, n
$$

(2) If $2 \leq m$,

$$
\theta_{\alpha} \leq u_{i} \leq P_{i} \theta_{\beta}, \quad i=1, \ldots, n
$$

where $\theta_{\gamma}$ is the unique positive solution (cf. [4]) of the problem

$$
\left\{\begin{aligned}
-\Delta z & =z^{1 / m}\left(\gamma-z^{1 / m}\right) & & \text { in } \Omega \\
z & =0 & & \text { on } \partial \Omega .
\end{aligned}\right.
$$

Proof. It is easy to see that if $u=\left(u_{1}, \cdots, u_{n}\right)$ is a positive solution of $(6)$, then it is a supersolution of (7) with $\gamma=\alpha$. On the other hand, there exists a positive subsolution $\underline{u}$ of (7) such that $\underline{u} \leq u$. So, $\theta_{\alpha} \leq u_{i}, i=1, \ldots, n$ by the uniqueness of positive solution of $(7)$.

Now, we consider the family

$$
u_{i r}=r^{m} P_{i} \theta_{\beta} . \quad i=1, \ldots, n \quad r \in\left(r_{0}, r_{1}\right) . \quad \text { with } r_{1}>r_{0}>1
$$

for some parameters $P_{i}$ to be chosen. To verify the hypotheses of Proposition 3.4 , we need that

$$
-\Delta u_{i r}-u_{i r}^{1 / m}\left(\lambda_{i}-u_{i r}^{1 / m}+\sum_{\substack{j=1 \\ j \neq i}}^{n} a_{i j} u_{j r}^{1 / m}\right) \geq 0, \quad i=1, \ldots, n .
$$

A straightforward calculation gives

$$
\begin{gathered}
-\Delta u_{i r}-u_{i r}^{1 / m}\left(\lambda_{i}-u_{i r}^{1 / m}+\sum_{\substack{j=1 \\
j \neq i}}^{n} a_{i j} u_{j r}^{1 / m}\right) \\
=r P_{i}^{1 / m} \theta_{\beta}^{1 / m}\left[\left(r^{m-1} P_{i}^{1-1 / m} \beta-\lambda_{i}\right)+\theta_{\beta}^{1 / m}\left(-r^{m-1} P_{i}^{1-1 / m}+r\left(P_{i}^{1 / m}-\sum_{\substack{j=1 \\
j \neq i}}^{n} a_{i j} P_{j}^{1 / m}\right)\right)\right] \\
\geq r P_{i}^{1 / m} \theta_{\beta}^{1 / m}\left[\left(r^{m-1} P_{i}^{1-1 / m}-1\right) \lambda_{i}+\theta_{\beta}^{1 / m}\left(-r^{m-1} P_{i}^{1-1 / m}+r\left(P_{i}^{1 / m}-\sum_{\substack{j=1 \\
j \neq i}}^{n} a_{i j} P_{j}^{1 / m}\right)\right)\right]
\end{gathered}
$$


for $i=1, \ldots, n$. Note that the linear system

$$
\begin{gathered}
P_{i}^{1 / m}-\sum_{\substack{j=1 \\
j \neq i}}^{n} a_{i j} P_{j}^{1 / m}=1, \quad i=1, \ldots, n \\
\Longleftrightarrow\left(\begin{array}{c}
P_{1}^{1 / m} \\
\vdots \\
P_{n}^{1 / m}
\end{array}\right)=\left(\begin{array}{cccc}
0 & a_{12} & \ldots & a_{1 n} \\
\vdots & \vdots & \vdots & \vdots \\
a_{n 1} & a_{n 2} & \ldots & 0
\end{array}\right)\left(\begin{array}{c}
P_{1}^{1 / m} \\
\vdots \\
P_{n}^{1 / m}
\end{array}\right)+\left(\begin{array}{c}
1 \\
\vdots \\
1
\end{array}\right)
\end{gathered}
$$

has a unique solution whose components are greater than 1 . Indeed, the iterative method tied to the former fixed point equation is convergent since $\rho(A)<1$; and, since the matrix is nonnegative, the convergent sequence to the solution which emanates from the origin has each component of each term greater than 1 . If we take these values as $P_{i}^{1 / m}, i=1, \ldots, n$, then $P_{i}>1, i=1, \ldots, n$. Hence,

$$
\begin{aligned}
& -\Delta u_{i r}-u_{i r}^{1 / m}\left(\lambda_{i}-u_{i r}^{1 / m}+\sum_{\substack{j=1 \\
j \neq i}}^{n} a_{i j} P_{j}^{1 / m}\right) \\
& \quad \geq r P_{i}^{1 / m} \theta_{\beta}^{1 / m}\left[\left(r^{m-1} P_{i}^{1-1 / m}-1\right) \lambda_{i}+\theta_{\beta}^{1 / m}\left(-r^{m-1} P_{i}^{1-1 / m}+r\right)\right] \text { for } i=1, \ldots, n .
\end{aligned}
$$

Case 1. If $1<m<2$, we take

$$
-r^{m-1}\left[\max _{1 \leq i \leq n}\left(P_{i}\right)\right]^{1-1 / m}+r \geq 0 \Longleftrightarrow r \geq r_{0}:=\left[\max _{1 \leq i \leq n}\left(P_{i}\right)\right]^{(m-1) /[m(2-m)]}>1 .
$$

The claim follows from Proposition 3.4.

Case 2. If $m \geq 2$, we have for each $i$

$$
r-r^{m-1} P_{i}^{1-1 / m}=r\left(1-r^{m-2} P_{i}^{1-1 / m}\right) \leq 0
$$

for whatever $r_{0} \geq 1$ we choose. So, since $\theta_{\beta}^{1 / m} \leq \beta$ (cf. [4]), we can apply Proposition 3.4 if

$$
\left(r^{m-1} P_{i}^{1-1 / m}-1\right) \lambda_{i}+\beta\left(-r^{m-1} P_{i}^{1-1 / m}+r\right) \geq 0 \text { for } i=1, \ldots, n,
$$

which is trivial, because

$$
\begin{aligned}
& \left(r^{m-1} P_{i}^{1-1 / m}-1\right) \lambda_{i}+\beta\left(-r^{m-1} P_{i}^{1-1 / m}+r\right) \\
\geq & \lambda_{i}\left(r^{m-1} P_{i}^{1-1 / m}-1-r^{m-1} P_{i}^{1-1 / m}+r\right) \geq 0
\end{aligned}
$$

with no new restriction for $r_{0}$, so that we can apply Proposition 3.4 with $r_{0}$ defined in (9). The proof finishes as in Case 1. 


\section{The parabolic problem.}

5.1. Case $\rho(A)<1$. Let $M_{i} i=1, \ldots, n$ denote the solution for the linear system

$$
M_{i}-\sum_{\substack{j=1 \\ j \neq i}}^{n} a_{i j} M_{j}=\lambda_{i}, \quad 1 \leq i \leq n,
$$

which verifies $M_{i}>\lambda_{i}, i=1, \ldots, n$ (previously, it was $P_{i}>1$ ). Observe that these $n$ numbers are independent of $m$.

Denote $\delta=\inf _{\bar{\Omega}} \frac{\theta_{\alpha}}{\theta_{\beta}}$ which satisfies $0<\delta \leq 1$ (cf. [4]). We have

Theorem 5.1. Suppose $\rho(A)<1$. If $\lambda_{i} \in \mathbf{R}_{+} \backslash\{0\}$ for $i=1, \ldots, n$, then there exists one unique global positive solution $u_{i}(t, x), \quad i=1, \ldots, n$ of the parabolic problem (2) and at least one positive solution $u_{s i}(x), \quad i=1, . ., n$ of the elliptic problem (6).

Moreover, if one of the following options holds:

(1) $1<m<2$ and

$$
\max _{1 \leq i \leq n} \sum_{\substack{j=1 \\ j \neq i}}^{n} a_{i j} P_{j}^{1 / m} \leq\left(\frac{\delta}{r_{0}}\right)^{1 / m}
$$

(2) $m=2$,

(3) $m>2$ and the values $M_{i}$ (solutions of (10)) are such that verify

$$
\lambda_{i} \geq \frac{m-2}{m-1} M_{i}, \quad i=1, \ldots, n,
$$

then $u_{s i}, \quad i=1, \ldots, n$ is the unique positive solution of $(6)$.

REMARK 5.2. Observe that, given $m>2$, it can occur that no n-tuple of nonnegative numbers $\lambda_{i}, \quad i=1, \ldots, n$ verify (11). However, given an n-tuple of nonnegative numbers with some of them positive, we can find a value of $m$ close to 2 for which (11) is true.

Proof. Assume that $\lambda_{i}>0$ for all $i=1, \ldots, n$. If $\lambda_{i}>0$ for some $i=1, \ldots, n$, the proof follows analogously. The argument of the proof is similar to Theorem 10 of [4] where the reader can find details.

The possible solutions of steady system (6) are bounded; in fact, from the maximum principle, it follows that

$$
\theta_{\lambda_{i}} \leq u_{s i} \leq M_{i}^{m}
$$

The sub-supersolution method gives the existence of solution for the steady problem. The same sub-supersolution of the steady problem is also a sub-supersolution of the parabolic problem independent of the time; this shows the existence of global solution for this problem. Finally, the uniqueness of positive solution for the parabolic problem follows from Proposition 3.3.

The uniqueness of solutions of the elliptic problem follows from Proposition 3.4. In fact, the a priori bounds of positive solutions (see (12)) allow us to obtain always one minimal positive solution which we denote $u_{* i}(x), \quad i=1, \ldots, n$. It will be enough that every solution $u_{s i}$ of this problem verifies

$$
u_{s i} \leq u_{* i}, \quad i=1, \ldots, n .
$$


For this, we consider the family of subsolutions $W_{r}=\left(r u_{s i}\right), \quad i=1, \ldots, n$ with $r \in[\underline{r}, 1)$ and $\underline{r}$ sufficiently small such that

$$
\underline{r} u_{s i} \leq u_{* i}, \quad i=1, \ldots, n .
$$

The conditions for $W_{r}$ are

$$
\lambda_{i}\left(1-r^{1-1 / m}\right)+\left(\sum_{\substack{j=1 \\ j \neq i}}^{n} a_{i j} u_{s j}^{1 / m}-u_{s i}^{1 / m}\right)\left(r^{1 / m}-r^{1-1 / m}\right) \geq 0 \quad \text { for } i=1, \ldots, n .
$$

(1) If $1<m<2$, since $r<1$, we have

$$
1-r^{1-1 / m}>0 \text { and } r^{1 / m}-r^{1-1 / m}<0 .
$$

In order to verify (13), it will be enough that

$$
\sum_{\substack{j=1 \\ j \neq i}}^{n} a_{i j} u_{s j}^{1 / m}-u_{s i}^{1 / m} \leq 0, \quad i=1, \ldots, n \text {. }
$$

By the estimates of Theorem 4.1, we have

$$
\sum_{\substack{j=1 \\ j \neq i}}^{n} a_{i j} u_{s j}^{1 / m} \leq \sum_{\substack{j=1 \\ j \neq i}}^{n} a_{i j}\left(r_{0} P_{j} \theta_{\beta}\right)^{1 / m} \leq \theta_{\alpha}^{1 / m} \leq u_{s i}^{1 / m}
$$

(2) If $m=2$, the inequalities are trivially true.

(3) If $m>2$, we have $r^{1 / m}>r^{1-1 / m}$ and by (12) the sufficient condition is

$$
\lambda_{i}\left(1-r^{1-1 / m}\right)-M_{i}\left(r^{1 / m}-r^{1-1 / m}\right) \geq 0, \quad i=1, \ldots, n .
$$

But the function

$$
f(r)=\frac{1-r^{1-1 / m}}{r^{1 / m}-r^{1-1 / m}}
$$

is decreasing for $r \leq 1$ and $\lim _{r \rightarrow 1}-f(r)=\frac{m-1}{m-2}$. So, (13) follows from the hypothesis of Theorem 5.1 .

The proof concludes as the one of Theorem 10 of [4].

5.2. Case $\rho(A)=1$. In this case, the following result can be stated.

TheOREm 5.3. Suppose that $\rho(A)=1$ and $m>1$. If $\lambda_{i} \in \mathbf{R}_{+} \backslash\{0\}$, for $i=1, \ldots, n$, then there exist one unique global positive solution of (2) and at least one positive solution of (6).

Proof. We will build a pair of sub-supersolutions for (2) and (6) independent of the time. As subsolution we can pick for each $i, u_{i}=\sigma \theta_{\alpha}$ with $\sigma>0$ a constant to choose. For the supersolution, we consider the function $\varphi(x)=R^{2}-|x|^{2}$ with $R$ large enough for $\varphi>0$ on $\bar{\Omega}$ and we look for the supersolution in the form

$$
\overline{u_{1}}=K \varphi, \quad \overline{u_{i}}=K k_{i} \varphi, \quad i=2, \ldots, n
$$


with $k_{i}>0, \quad i=2, \ldots, n$ and $K>0$ to be chosen. We must verify

$$
\left\{\begin{aligned}
2 K N-(K \varphi)^{1 / m}\left(\lambda_{1}+(K \varphi)^{1 / m}\left(-1+\sum_{j=2}^{n} a_{1 j} k_{j}^{1 / m}\right)\right) & \geq 0 \\
2 K k_{i} N-\left(K k_{i} \varphi\right)^{1 / m}\left(\lambda_{i}+(K \varphi)^{1 / m}\left(-k_{i}^{1 / m}+a_{i 1}+\sum_{\substack{j=2 \\
j \neq i}}^{n} a_{i j} k_{j}^{1 / m}\right)\right) & \geq 0
\end{aligned}\right.
$$

for $i=2, \ldots, n$. These inequalities hold if $K$ is sufficiently large and we find positive $k_{i}$ such that

$$
\left\{\begin{aligned}
-1+\sum_{\substack{j=2 \\
n}}^{n} a_{1 j} k_{j}^{1 / m} & =0 \\
-k_{i}^{1 / m}+a_{i 1}+\sum_{\substack{j=2 \\
j \neq i}}^{n} a_{i j} k_{j}^{1 / m} & =0
\end{aligned}\right.
$$

for $i=2, \ldots, n$. It is easy to check that the compatibility condition of $(14)$ is $\rho(A)=1$. On the other hand, the system of the $n-1$ last equations of (14) can be written as

$$
\left(\begin{array}{c}
k_{2}^{1 / m} \\
\vdots \\
k_{n}^{1 / m}
\end{array}\right)=\left(\begin{array}{cccc}
0 & a_{23} & \ldots & a_{2 n} \\
\vdots & \vdots & \vdots & \vdots \\
a_{n 2} & a_{n 3} & \ldots & 0
\end{array}\right)\left(\begin{array}{c}
k_{2}^{1 / m} \\
\vdots \\
k_{n}^{1 / m}
\end{array}\right)+\left(\begin{array}{c}
a_{21} \\
\vdots \\
a_{n 1}
\end{array}\right)
$$

The matrix of this system has spectral radius $<1$ (cf. Theorem 2.2 ), and so the solution is positive.

5.3. Case $\rho(A)>1$. Suppose that in (2) we fix $i$, we let $a_{i j}=0, j=1, \ldots, n ; i \neq j$, and we leave equation $i$ out. We start proving that if the resulting $n$-1-dimensional system has solutions that blow up in finite time, then the solutions of (2) also blow up in finite time. We will suppose without loss of generality that $i=n$.

TheOREm 5.4. Suppose that the solutions of the problem

$$
\left\{\begin{array}{llll}
\frac{\partial}{\partial t} u_{i}-\Delta u_{i} & =u_{i}^{1 / m}\left(\lambda_{i}-u_{i}^{1 / m}+\sum_{\substack{j=1 \\
j \neq i}}^{n-1} a_{i j} u_{j}^{1 / m}\right) & & \text { in } D_{T}, \\
u_{i} & =0 & & \\
u_{i}(0, x) & =u_{i}^{0}(x) & \text { in } S_{T}, & \\
& \text { on } \bar{\Omega}, &
\end{array}\right.
$$

blow up in finite time. Then the solutions of (2) blow up also in finite time whenever $a_{\text {in }}>0$.

Proof. It will be enough to check that $(\underline{u}, \bar{u})=(0, u)$ is a sub-supersolution of $(15)$, where $u=\left(u_{1}, \cdots, u_{n-1}\right)$ and $\left(u_{1}, \cdots, u_{n}\right)$ is one solution of the system (2). The uniqueness of positive solution of (15) implies that

$$
0 \leq \tilde{u}_{i} \leq u_{i}, \quad i=1, \ldots, n-1
$$

where $\tilde{u}_{i}$ is the solution of (15). The result follows. 
So, if a subsystem of dimension lower than $n$ blows up in finite time, then the $n$ dimensional system also blows up in finite time. So we can suppose the following hypothesis:

$$
\left\{\begin{array}{l}
\text { Given (2), the solutions of any system of dimension } \leq n-1 \\
\text { extracted from it, are global in time for any } \lambda_{i}>0
\end{array}\right.
$$

since otherwise the blow-up in finite time will be sure. Note that the matrix of a subsystem is a principal submatrix of $A$.

To justify the main result we consider the function

$$
f(r)=r^{2-m}+a_{1 n} r-a_{n 1} r^{1-m}-1 .
$$

It is easy to prove that if $1<m<2$,

$$
\lim _{r \downarrow 0^{+}} f(r)=-\infty, \quad \lim _{r \uparrow+\infty} f(r)=+\infty, \quad f^{\prime}(r)>0 \quad \text { if } r>0
$$

and therefore the equation $f(r)=0$ has one positive solution which we call $\bar{r}$.

The main result is the following

Theorem 5.5. Suppose that $\rho(A)>1$ and $1<m<2$. Then, there exist $\overline{\lambda_{i}}>0, \quad i=$ $1, \ldots, n$ such that for $\lambda_{i}>\overline{\lambda_{i}}$, the solution of (2) blows up in finite time.

Proof. There is nothing to prove if $(\mathrm{H})$ is not verified. So, suppose $(\mathrm{H})$. We use the induction on $\operatorname{dim}(A)=n$. The result is true for $n=2$ (cf. [4]). Suppose the result is also true until $n-1$; in other words, suppose that if the associated matrix to a system with dimension lower than $n-1$ has the spectral radius $>1$, then its solutions blow up in finite time. (4) ensures that if for any principal submatrix $A_{i}$, $\operatorname{det}\left(I-A_{i}\right)<0$, then $\rho\left(A_{i}\right)>1$ and, for the induction hypothesis, $(\mathrm{H})$ is not true; so, every principal submatrix of $A, A_{i}$, must verify $\operatorname{det}\left(I-A_{i}\right) \geq 0$. And, by Lemma 2.1 , it will be true also that $\operatorname{det}(I-A)<0$.

For the case $n$, the proof is carried out in two steps.

Step 1

Suppose that

$$
\lambda_{n}=\bar{r}^{m-1} \lambda_{1} ; \quad a_{n j}=\bar{r}^{m-1} a_{1 j}, \quad j=2, \ldots, n-1 ; \quad u_{n}^{0}(x)=\bar{r}^{m} u_{1}^{0}(x) .
$$

We search the conditions which ensure that the solution of (2) is of the form $u_{n}=k u_{1}$ for some $k>0$. It is not difficult to show that (2) becomes

$$
\left\{\begin{array}{lll}
\frac{\partial}{\partial t} u_{1}-\Delta u_{1} & =u_{1}^{1 / m}\left(\lambda_{1}-\left(1-a_{1 n} k^{1 / m}\right) u_{1}^{1 / m}+\sum_{j=2}^{n-1} a_{i j} u_{j}^{1 / m}\right) & \\
\frac{\partial}{\partial t} u_{i}-\Delta u_{i} & =u_{i}^{1 / m}\left(\lambda_{i}-u_{i}^{1 / m}+\left(a_{i 1}+a_{i n} k^{1 / m}\right) u_{1}^{1 / m}+\sum_{\substack{j=2 \\
j \neq i}}^{n-1} a_{i j} u_{j}^{1 / m}\right) & \\
& & \text { in } D_{T}, \\
\frac{\partial}{\partial t} u_{1}-\Delta u_{1} & =k^{1 / m-1} u_{1}^{1 / m}\left(\lambda_{n}-\left(k^{1 / m}-a_{n 1}\right) u_{1}^{1 / m}+\sum_{j=2}^{n-1} a_{n j} u_{j}^{1 / m}\right) & \\
u_{i} & =0 & \text { in } D_{T}, \\
u_{i}(0, x) & =u_{i}^{0}(x) & \text { in } S_{T}, \\
& & \text { on } \bar{\Omega},
\end{array}\right.
$$


for $i=2, . ., n-1$. The first and the last equations are the same if $k=\bar{r}^{m}$, in which case the system comes down to an $n$-1-dimensional one which has the following matrix:

$$
\mathcal{A}_{1}=\left(\begin{array}{cccc}
-\left(1-a_{1 n} \bar{r}\right) & a_{12} & \ldots & a_{1, n-1} \\
a_{21}+a_{2 n} \bar{r} & -1 & \ldots & a_{2, n-1} \\
\vdots & \vdots & \ddots & \vdots \\
a_{n-1,1}+a_{n-1, n} \bar{r} & a_{n-1,2} & \ldots & -1
\end{array}\right)
$$

and whose associated matrix is (see $(3))$

$$
A_{1}=\left(\begin{array}{cccc}
0 & c_{12} & \ldots & c_{1, n-1} \\
c_{21} & 0 & \ldots & a_{2, n-1} \\
\vdots & \vdots & \ddots & \vdots \\
c_{n-1,1} & a_{n-1,2} & \ldots & 0
\end{array}\right)
$$

being

$$
c_{1 j}=a_{1 j}\left(1-a_{1 n} \bar{r}\right)^{(m-1) /(2-m)}, \quad c_{i 1}=\left(a_{i 1}+a_{i n} \bar{r}\right)\left(1-a_{1 n} \bar{r}\right)^{1 /(m-2)}
$$

for $i, j=2, \ldots, n-1$. Note that

$$
1-a_{1 n} \bar{r}>0 .
$$

Indeed, it is trivial to check that

$$
f\left(\frac{1}{a_{1 n}}\right)=\left(\frac{1}{a_{1 n}}\right)^{1-m}\left(\frac{1-a_{1 n} a_{n 1}}{a_{1 n}}\right)>0
$$

since the principal minor of $I-A$ generated by the submatrix

$$
B=\left(\begin{array}{cc}
1 & -a_{1 n} \\
-a_{n 1} & 1
\end{array}\right)
$$

is positive. Indeed, if $\rho(I-B)=1$, then the spectral radius of any submatrix containing $I-B$ will be greater than 1 (see Theorem 2.2), and the induction hypothesis and Theorem 5.4 complete the proof. By Theorem 5.4 and the induction hypothesis it remains to prove that $\rho\left(A_{1}\right)>1$. And, by (4), it will be sufficient to justify that $\operatorname{det}\left(I-A_{1}\right)<0$. We know that

$$
\operatorname{det}(I-A)=\left|\begin{array}{cccc}
1 & -a_{12} & \ldots & -a_{1 n} \\
-a_{21} & 1 & \ldots & -a_{2 n} \\
\vdots & \vdots & \ddots & \vdots \\
-a_{n 1} & -a_{12} \bar{r}^{m-1} & \ldots & 1
\end{array}\right|<0
$$

Then, through elementary transformations (we change the first row for the sum of such row and the product of the row $n$ multiplied by $-\bar{r}^{1-m}$, we multiply the first row by $1 /\left(a_{1 n}+\bar{r}^{1-m}\right)$ and the column $n$ by $\bar{r}$ and we change column 1 by the sum of the columns 
1 and $n$ ) and developing by the first row, we obtain

$$
\begin{gathered}
\operatorname{det}(I-A)=\left|\begin{array}{cccc}
1 & -a_{12} & \ldots & -a_{1 n} \\
-a_{21} & 1 & \ldots & -a_{2 n} \\
\vdots & \vdots & \ddots & \vdots \\
-a_{n 1} & -a_{12} \bar{r}^{m-1} & \ldots & 1
\end{array}\right| \\
=\left(\bar{r}^{1-m}+a_{1 n}\right)(-1)^{n+2}\left|\begin{array}{cccc}
-a_{21}-a_{2 n} \bar{r} & 1 & \ldots & -a_{2, n-1} \\
-a_{31}-a_{3 n} \bar{r} & -a_{32} & \ldots & -a_{3, n-1} \\
\vdots & \vdots & \ddots & \vdots \\
-a_{n 1}+\bar{r} & -a_{12} \bar{r}^{m-1} & \ldots & -a_{1, n-1} \bar{r}^{m-1}
\end{array}\right| .
\end{gathered}
$$

Now we pass the last row to the first one, multiply the first row by $p \bar{r}^{1-m}, \quad p:=$ $\left(1-a_{1 n} \bar{r}\right)^{(1-m) /(m-2)}$ and the first column by $q:=\left(1-a_{1 n} \bar{r}\right)^{1 /(m-2)}$, and we obtain

$$
\begin{gathered}
\operatorname{det}(I-A)=\left|\begin{array}{cccc}
1 & -a_{12} & \ldots & -a_{1 n} \\
-a_{21} & 1 & \ldots & -a_{2 n} \\
\vdots & \vdots & \ddots & \vdots \\
-a_{n 1} & -a_{12} \bar{r}^{m-1} & \ldots & 1
\end{array}\right| \\
=\frac{\bar{r}^{1-m}+a_{1 n}}{p q \bar{r}^{1-m}}(-1)^{2 n}\left|\begin{array}{cccc}
\left(-a_{n 1}+\bar{r}\right) \bar{r}^{1-m} p q & -a_{12} p & \ldots & -a_{1, n-1} p \\
\left(-a_{21}-a_{2 n} \bar{r}\right) q & 1 & \ldots & -a_{2, n-1} \\
\vdots & \vdots & \ddots & \vdots \\
\left(-a_{n-1,1}-a_{n-1, n} \bar{r}\right) q & -a_{n-1,2} & \ldots & 1 \\
=\frac{\bar{r}^{1-m}+a_{1 n}}{p q \bar{r}^{1-m}} \operatorname{det}\left(I-A_{1}\right) & &
\end{array}\right|
\end{gathered}
$$

since $\left(-a_{n 1}+\bar{r}\right) \bar{r}^{1-m} p q=1$. It follows that $\operatorname{det}\left(I-A_{1}\right)<0$, as we needed.

Step 2

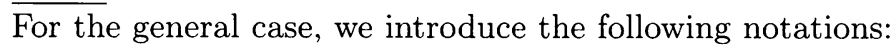

$$
\left\{\begin{array}{l}
\text { If } a_{n j} \geq \bar{r}^{m-1} a_{1 j}, \text { then } d_{1 j}:=a_{1 j}, \quad d_{n j}:=\bar{r}^{m-1} a_{1 j} \\
\text { If } a_{n j}<\bar{r}^{m-1} a_{1 j}, \text { then } d_{1 j}:=\bar{r}^{1-m} a_{n j}, \quad d_{n j}:=a_{n j}
\end{array} \quad j=1, \ldots, n .\right.
$$

We pose now the problem

$$
\left\{\begin{array}{lll}
\frac{\partial}{\partial t} v_{1}-\Delta v_{1} & =v_{1}^{1 / m}\left(\bar{r}^{1-m} \lambda_{n}-v_{1}^{1 / m}+\sum_{j=2}^{n} d_{1 j} v_{j}^{1 / m}\right) & \\
\frac{\partial}{\partial t} v_{i}-\Delta v_{i} & =v_{i}^{1 / m}\left(\lambda_{i}-v_{i}^{1 / m}+\sum_{\substack{j=1 \\
j \neq i}}^{n} a_{i j} v_{j}^{1 / m}\right) & \\
\frac{\partial}{\partial t} v_{n}-\Delta v_{n} & =v_{n}^{1 / m}\left(\lambda_{n}-v_{n}^{1 / m}+\sum_{j=1}^{n-1} d_{n j} v_{j}^{1 / m}\right) & \\
v_{i} & =0 & \text { in } D_{T} \\
v_{i}(0, x) & =v_{i}^{0}(x) & \text { in } S_{T} \\
& & \text { on } \bar{\Omega}
\end{array}\right.
$$


for $i=2, \ldots, n-1$, defining $v_{1}^{0}=\bar{r}^{-m} v_{n}^{0}, \quad v_{i}^{0}=u_{i}^{0}, 1=2, \ldots, n-1$ and choosing $v_{n}^{0} \in$ int $(P)$ and such that $v_{n}^{0}<\bar{r}^{m} u_{1}^{0}$. This problem is in the framework of Step 1 and its solutions bound from below the ones of the original problem if $\lambda_{1}>\bar{r}^{1-m} \lambda_{n}$, from where the conclusion follows. A similar reasoning can be used when $\lambda_{1}<\bar{r}^{1-m} \lambda_{n}$.

However, we can obtain global solution when the parameters $\lambda_{i}$ are small enough or when the diffusion is slow enough.

TheOrem 5.6. Suppose $(\mathrm{H})$ and $\rho(A)>1$. Then

(1) If $1<m<2$, then there exist values $\underline{\lambda_{i}}>0$ such that for $0<\lambda_{i}<\underline{\lambda_{i}}$, there is one unique global positive solution of (2) and at least one positive solution of the corresponding steady problem.

(2) If $2<m$, then whenever $\lambda_{i}>0$, there is one unique global positive solution of (2) and at least one positive solution of the corresponding steady problem.

Proof. By the sub-supersolutions method, we look for the supersolution in the form $\overline{u_{i}}=S \psi, \psi$ being the positive solution of

$$
\left\{\begin{array}{rll}
-\Delta w & =1 & \text { in } \Omega \\
w & =0 & \text { on } \partial \Omega,
\end{array}\right.
$$

and $S>0$ a positive constant to choose. The $n$-tuple $\overline{u_{i}}$ is a supersolution of $(2)$ if

$$
S^{1-1 / m}+S^{1 / m} \psi^{2 / m}\left(1-\sum_{\substack{j=1 \\ j \neq i}}^{n} a_{i j}\right) \geq \lambda_{i} \psi^{1 / m}, \quad i=1, \ldots, n
$$

If $1<m<2,(17)$ can be written as

$$
S^{1-1 / m}\left(1+S^{2 / m-1} \psi^{2 / m}\left(1-\sum_{\substack{j=1 \\ j \neq i}}^{n} a_{i j}\right)\right) \geq \lambda_{i} \psi^{1 / m}, \quad i=1, \ldots, n .
$$

The inequalities (18) hold provided $\lambda_{i}$ is chosen sufficiently small or even whenever $\lambda_{i}$ under a stronger condition than $(\mathrm{H}), 1-\sum_{\substack{j=1 \\ j \neq i}}^{n} a_{i j} \geq 0$.

If $2<m,(17)$ can be written as

$$
S^{1 / m}\left(S^{1-2 / m}+\psi^{2 / m}\left(1-\sum_{\substack{j=1 \\ j \neq i}}^{n} a_{i j}\right)\right) \geq \lambda_{i} \psi^{1 / m}, \quad i=1, \ldots, n,
$$

and are satisfied whenever $\lambda_{i}>0$ if $S$ is sufficiently large.

We look for the subsolutions in the form $\underline{u}_{i}=\sigma \theta_{\alpha}$ with $\sigma>0$ constant to choose. The conditions to verify are

$$
\left(\sigma^{1-1 / m}-1\right) \alpha-\theta_{\alpha}^{1 / m}\left(\sigma^{1-1 / m}+\sigma^{1 / m}\left(-1+\sum_{\substack{j=1 \\ j \neq i}}^{n} a_{i j}\right)\right) \leq 0, \quad i=1, \ldots, n,
$$

that can be obtained doing $\sigma$ sufficiently small.

Finally, the pair of sub-supersolutions can be ordered.

In the case $m=2$, we can prove 
Theorem 5.7. Suppose $(\mathrm{H}), \rho(A)>1$ and $m=2$. Then (2) has one unique global positive solution $u=\left(u_{1}, \cdots, u_{n}\right)$. In this case, it can occur that $\left\|u_{i}(x, t)\right\|_{\infty} \rightarrow \infty$ as $t \rightarrow+\infty$ for some $i=1, \cdots, n$.

Proof. It suffices to use as sub-supersolutions

$$
\overline{u_{i}}=K e^{\gamma t}, \quad \underline{u_{i}}=\sigma \theta_{\alpha}, \quad i=1, \ldots, n,
$$

$K, \gamma$, and $\sigma$ being suitable positive constants.

6. The case $m=1$. In the case $m=1$, the above results can be clarified. In fact, we can prove the following result, which generalizes Theorems 3.1, 3.2, and 3.3 in [7] and Theorem 3.2 in [6]. We need the following notation. We define

$$
b_{i i}=0, \quad b_{i j}=a_{i j} \quad \text { for } i \neq j, \quad 1 \leq i, j \leq n
$$

and let

$$
A=\left(b_{i j}\right) .
$$

Let $\sigma_{1}$ and $\phi_{1}$ be the principal eigenvalue and the corresponding positive eigenfunction of the laplacian with homogeneous Dirichlet boundary conditions. We denote $w_{\gamma}$ the unique positive solution of (7) with $m=1$, which exists if, and only if, $\gamma>\sigma_{1}$. Moreover, we denote $\alpha=\min _{1 \leq i \leq n} \lambda_{i}, \beta=\max _{1 \leq i \leq n} \lambda_{i}$ and, if $\alpha>\sigma_{1}$,

$$
\rho:=\inf _{\bar{\Omega}} \frac{w_{\alpha}}{w_{\beta}}
$$

It is well known that $0<\rho \leq 1$. Finally, we will write $Q_{i} i=1, \ldots, n$ to denote the solution of (8) with $m=1$.

Theorem 6.1. Assume $\alpha>\sigma_{1}$. Then,

(1) Problem (6) with $m=1$ possesses one positive solution if and only if $\rho(A)<1$. In this case, for any positive solution $u_{i}$ of (6) for $m=1$, it holds:

$$
w_{\alpha} \leq u_{i} \leq Q_{i} w_{\beta}, \quad i=1, \ldots, n .
$$

(2) If $\rho(A)<1$, there exists one unique global positive solution $u_{i}(t, x), \quad i=1, \ldots, n$ of the parabolic problem (2) for $m=1$ and at least one positive solution $u_{s i}(x), \quad i=1, . ., n$ of the elliptic problem (6) for $m=1$.

Moreover, if

$$
\max _{1 \leq i \leq n} \sum_{\substack{j=1 \\ j \neq i}}^{n} a_{i j} Q_{j} \leq \rho
$$

holds, then $u_{s i}, \quad i=1, \ldots, n$ is the unique positive solution of $(6)$ for $m=1$.

(3) If $\rho(A)=1$, there exists one unique positive global solution of (2) for $m=1$, and as $t \rightarrow \infty$,

$$
\left\|u_{i}(x, t)\right\|_{\infty} \rightarrow \infty, \quad \text { for all } i=1, \ldots, n .
$$

(4) If $\rho(A)>1$, the positive solution of (2) for $m=1$ blows up in finite time. 
Proof. Assume $\rho(A)<1$. The existence of positive solution of $(6)$ with $m=1$, (19) and the second paragraph follow by Theorems 4.1 and 5.1 .

Now, on the contrary, suppose that there exists one positive solution $u_{i}, i=1, \ldots, n$, of (6) with $m=1$ and that $\rho(A) \geq 1$. Let $\hat{\alpha}=\left(\alpha_{1}, \ldots, \alpha_{n}\right)$ be the componentwise positive eigenvector of $A$ associated to $\rho(A)$, i.e.,

$$
A \hat{\alpha}=\rho(A) \hat{\alpha} .
$$

Take $W_{r}=\left(r \alpha_{i} \phi_{1}\right), i=1, \ldots, n$ with $r \in\left[r_{0}, r_{1}\right)$ with $r_{0}$ sufficiently small such that

$$
r_{0} \alpha_{i} \leq u_{i} \quad i=1, \ldots, n \text {. }
$$

Observe that $W_{r}$ is a family of subsolutions. Indeed, for each $i=1, \ldots, n$ we have

$$
\begin{aligned}
-\Delta\left(r \alpha_{i} \phi_{1}\right)-\left(r \alpha_{i} \phi_{1}\right)\left(\lambda_{i}-r \alpha_{i} \phi_{1}+\sum_{\substack{j=1 \\
j \neq i}}^{n} a_{i j} r \alpha_{j} \phi_{1}\right) & \\
& =r \alpha_{i} \phi_{1}\left(\sigma_{1}-\lambda_{i}+r \phi_{1} \alpha_{i}(1-\rho(A))\right) \leq 0 .
\end{aligned}
$$

So, $W_{r}$ is subsolution for all $r>r_{0}$. This shows the contradiction.

Suppose $\rho(A)=1$. Since $\alpha>\sigma_{1}$, it is easy to prove that $\left(\epsilon \phi_{1}, \ldots, \epsilon \phi_{1}\right)$ is a subsolution of (6) with $m=1$, provided that $\epsilon$ is sufficiently small. Hence, the positive solution, $u_{i}(t, x)$ of $(2)$ with $m=1$ and $u_{i}^{0}=\epsilon \phi_{1}$, is monotone nondecreasing in $t$ (see Lemma 10.4.1 in [10] and Lemma 3.1 in [6], for instance). If $u_{i}$ is bounded in $\|\cdot\|_{\infty}$ for all $i=1, \ldots, n$, then $u_{i}$ converges to a positive solution of (6) with $m=1$, which is impossible by the first paragraph. So that, $u_{i}$ is unbounded for some $i$, and by Theorem 5.4 , the result follows.

Suppose $\rho(A)>1$. Firstly, observe that the function $f$ defined in (16) possesses one unique positive solution given by

$$
\bar{r}=\frac{1+a_{n 1}}{1+a_{1 n}} .
$$

In this case, we can repeat exactly the proof of Theorem 5.5, using in the induction process for $n=2$ Theorem 3.2 of $[6]$.

\section{REFERENCES}

[1] F. Ayres Jr., Matrices, McGraw-Hill, México, 1969.

[2] R. dal Passo and P. de Mottoni, Some existence, uniqueness and stability results for a class of semilinear degenerate elliptic systems, Boll. Un. Mat. Ital. C (6). 3, 203-231 (1975).

[3] M. Delgado, J. López-Gómez, and A. Suárez, On the symbiotic Lotka-Volterra model with diffusion and transport effects, J. Differential Eqns. 160, 175-262 (2000).

[4] M. Delgado and A. Suárez, Stability and uniqueness for cooperative degenerate Lotka-Volterra model, Nonlinear Analysis, 49, 757-778 (2002).

[5] M. Delgado and A. Suárez, Sistemas cooperativos de tipo Volterra-Lotka con tres especies, XVII Congress on Differential Equations and Applications/VII Congress on Applied Mathematics, Vol. I, II (Spanish) (Salamanca, 2001).

[6] P. Korman, Dynamics of the Lotka-Volterra systems with diffusion, Appl. Anal. 44, 191-207 (1992).

[7] P. Korman and A. Leung, On the existence and uniqueness of positive steady states in the VolterraLotka ecological models with diffusion, Appl. Anal. 26, 145-160 (1987).

[8] Y. Lou, Necessary and sufficient condition for the existence of positive solutions of certain cooperative system, Nonlinear Analysis, 26, 1079-1095 (1996). 
[9] P. J. McKenna and W. Walter, On the Dirichlet problem for elliptic systems, Appl. Anal. 21, 207-224 (1986).

[10] C. V. Pao, Nonlinear parabolic and elliptic equations, Plenum Press, New York, 1992.

[11] R. S. Varga, Matrix iterative analysis, Springer, Berlin, 2000. 